\title{
Diacronie
}

Studi di Storia Contemporanea

$N^{\circ} 27,3$ | 2016

Stato, costituzione e democrazia

\section{Un colpo di stato mancato? Il golpe Borghese e l'eversione nera in Italia}

Nicola Tonietto

\section{Q OpenEdition \\ 1 Journals}

Edizione digitale

URL: https://journals.openedition.org/diacronie/4247

DOI: $10.4000 /$ diacronie.4247

ISSN: 2038-0925

Editore

Association culturelle Diacronie

Notizia bibliografica digitale

Nicola Tonietto, «Un colpo di stato mancato? II golpe Borghese e l'eversione nera in Italia», Diacronie [Online], $N^{\circ} 27,3$ | 2016, documento 3, online dal 29 septembre 2016, consultato il 21 septembre 2021. URL: http://journals.openedition.org/diacronie/4247 ; DOI: https://doi.org/10.4000/diacronie.4247 


\title{
Diacronie
}

\section{Un colpo di stato mancato?}

\section{Il golpe Borghese e l'eversione nera in Italia}

\author{
Nicola TONIETTO *
}

Il golpe Borghese e l'eversione nera in Italia Il tentativo eversivo di Junio Valerio Borghese si inserisce nel quadro della strategia della tensione e della stagione stragista vissuta in Italia dal 1969 al 1974. Questo intervento mira a dimostrare come la minaccia di un rovesciamento dello Stato fosse reale. Grazie al materiale raccolto dalle inchieste giudiziarie possiamo delineare la ramificazione delle organizzazioni eversive: elementi legati all'estrema destra, ufficiali dell'esercito, esponenti della massoneria e della criminalità mafiosa. Ma soprattutto possiamo mostrare come, nonostante il fallimento dell'operazione, l'obiettivo del golpe (il mantenimento dell'Italia in una posizione anticomunista, centrista e filoatlantica) venga raggiunto senza che si renda utile un colpo di Stato.

\section{Introduzione}

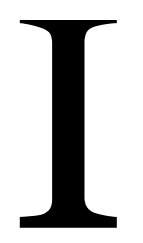

1 tentativo eversivo di Junio Valerio Borghese si inserisce nel quadro della strategia della tensione e più in particolare si collega con la stagione stragista vissuta in Italia dal 1969 al 1974. Si tratta di un periodo tragico ma allo stesso tempo determinante per la storia italiana, il quale influenzò e segnò pesantemente le complicate vicende politiche del nostro Paese. Questo saggio si prefigge lo scopo di evidenziare come la minaccia di un rovesciamento dello Stato fosse reale, un pericolo compreso anche dagli stessi politici che rivestivano, allora, le cariche più alte della Repubblica. Documenti e testimonianze tra di loro convergenti hanno

${ }^{1}$ Per quanto riguarda la strage della stazione di Bologna avvenuta nel 1980, nonostante una certa affinità con i precedenti episodi, lo scopo non è quello di essere utile per un eventuale colpo di stato. 
permesso di ricostruire queste vicende, e in particolare il tentato golpe Borghese, anche se rimane ancora qualche punto oscuro. Ciò che risulta chiaro è la ramificazione delle organizzazioni eversive sviluppatesi tra il 1969 e il 1974, l'appartenenza ad esse di elementi legati alla destra extraparlamentare (Fronte Nazionale, Ordine Nuovo, Avanguardia Nazionale), di ufficiali, anche di grado elevato, dell'esercito, di esponenti della massoneria e della criminalità mafiosa. Così come la conoscenza, da parte dei vertici dei servizi di sicurezza italiani (SID, Ufficio Affari Riservati), di alcuni politici italiani, nonché dei servizi, del personale dell'ambasciata e di politici di spicco statunitensi delle trame in atto nel nostro Paese. Si è cercato soprattutto di discernere tutte le forze in gioco, i gruppi di potere, le ambiguità che si celavano dietro un tentativo che, a prima vista, pareva del tutto velleitario.

Analizzare il periodo in cui si inserisce il golpe Borghese è un ulteriore modo per comprendere l'infondatezza della visione secondo la quale qualsiasi tentativo di colpo di stato elaborato in Italia avrebbe dovuto essere bollato come velleitario. Questo è il tragico bilancio di un quinquennio, 1969-1974, che ha insanguinato la storia della nostra Repubblica: 92 morti, 2795 feriti, 4065 attentati, fra cui 7 stragi ${ }^{2}$. Dati che non hanno eguali in nessun paese dell'Occidente3. Tutte le stragi in questione sono attribuibili all'estrema destra con, tuttavia, una rilevante differenza: da Piazza Fontana alla Questura di Milano erano finalizzate a far ricadere le responsabilità di esse sui gruppi di estrema sinistra, al contrario gli ultimi due casi (Piazza della Loggia e Italicus) erano dichiaratamente azioni di destra con finalità terroristiche.

La stagione delle stragi e dei tentativi eversivi prese il via con la crisi dell'esperienza dei governi di centrosinistra, nata sia con la speranza di aprire un'epoca riformista per la giovane democrazia italiana ma anche con l'intento di realizzare l'isolamento del PCI per mezzo della nascita di un grande partito socialdemocratico, il PSU, esperimento tentato con l'unificazione PSI-PSDI del 1966. Entrambi gli obiettivi furono fallimentari e produssero effetti opposti a quelli sperati: una netta avanzata elettorale del PCI alle elezioni del 1968 e l'attivazione della più intensa mobilitazione sociale della storia repubblicana. Il risultato fu una forte instabilità politica per il nostro Paese, il quale si trovava in una situazione di difficile risoluzione. Non era chiaro se si sarebbe abbandonata l'esperienza del centro-sinistra a favore di una soluzione centrista o di

\footnotetext{
2 Dati elaborati in CUCCHIARELLI, Paolo, GIANNULI, Aldo, Lo Stato parallelo. L'Italia “oscura” nei documenti e nelle relazioni della Commissione Stragi, Roma, Gamberetti, 1997, p. 370.

3 Vedi anche i dati del TWEED Dataset riportati in FUMIAN, Carlo, La storia. Alle armi, in CALOGERO, Pietro, FUMIAN, Carlo, SARTORI, Michele, Terrore rosso. Dall'autonomia al partito armato, Roma-Bari, Laterza, 2010, pp. 180-182.
} 
centro-destra o se ci sarebbe stata una maggiore apertura a sinistra verso il Partito Comunista4. Lo stesso contesto internazionale contribuiva ad aumentare la tensione: oltre alle proteste studentesche divampate negli Stati Uniti e nell'Europa Occidentale, si era assistito all'invasione sovietica della Cecoslovacchia, all'acuirsi della guerra in Vietnam, nonché ai conflitti in Africa, America Latina e Medio Oriente. L'estrema destra italiana, nonché elementi appartenenti alle Forze Armate, con l'influenza dei militari francesi e statunitensi, avevano elaborato la teoria della "guerra rivoluzionaria" 5 . Questa guerra non ortodossa praticata dalle forze comuniste avrebbe dovuto essere fatta propria anche dagli elementi di destra proprio per contrastare la loro avanzata. La strategia in questione, tuttavia, non era sufficiente: si rendeva necessario creare nell'opinione pubblica, mediante disordini e attentati da attribuire ai gruppi di estrema sinistra, quella richiesta d'ordine e fermezza necessaria a provocare l'intervento dell'esercito per eliminare la sovversione comunista ${ }^{6}$.

\section{Il Fronte Nazionale e la preparazione del golpe}

In questo contesto, il 13 settembre 1968 venne fondato a Roma il Fronte Nazionale. La nuova organizzazione di estrema destra era capeggiata da Junio Valerio Borghese, ex

\footnotetext{
4 PANVINI, Guido, «La pianificazione della violenza (1969-1972)», in VENTRONE, Angelo (a cura di), I dannati della rivoluzione. Violenza politica e storia d'Italia negli anni Sessanta e Settanta, Macerata, Edizioni Università di Macerata, 2010, p. 61.

5 È significativo che al noto convegno organizzato dall'Istituto Pollio dal 3 al 5 maggio 1965 avente come tema la "guerra rivoluzionaria", fossero presenti numerosi protagonisti delle trame eversive del decennio seguente, tra i quali Guido Giannettini, Pino Rauti, Pio Filippani Ronconi, Stefano Delle Chiaie, Mario Merlino. Vedi anche PANVINI, Guido, La destra eversiva, in ORSINA, Giovanni (a cura di), Storia delle destre nell'Italia repubblicana, Soveria Mannelli, Rubbettino, 2014, pp. 213-216.

6 Per un approfondimento sulla storia della "strategia della tensione" vedi DONDI, Mirco, L'eco del boato. Storia della strategia della tensione 1965-1974, Roma-Bari, Laterza, 2015; mentre per una storia del MSI e delle destre vedi, tra i contributi più recenti, CONTI, Davide, L'anima nera della Repubblica. Storia del MSI, Roma-Bari, Laterza, 2013; ORSINA Giovanni (a cura di), Storia delle destre nell'Italia repubblicana, Soveria Mannelli, Rubbettino, 2014.
} 
comandante della Decima Mas durante la Repubblica Sociale Italiana7. La base iniziale erano i soci del "Circolo Selvatici" di Roma e gli ex militari della RSI

Le posizioni del nuovo movimento erano esemplificate negli "Orientamenti programmatici” pubblicati nel gennaio dell'anno seguente. Per gli aderenti al Fronte era necessario costituire uno Stato forte, adottando un sistema di governo autorevole ed efficiente che si esprimesse nel quadro degli interessi nazionali. Era necessaria inoltre l'«esclusione dei partiti da ogni partecipazione all'attività del governo», così come la loro «esclusione dal sistema sindacale» e la successiva «costituzione di associazioni di categorie unitarie con dirigenti di provata competenza professionale»9. Per quanto riguardava la politica interna, essa doveva essere «intesa alla coesione nazionale, all'osservanza rigorosa delle leggi, alla difesa dell'ordine pubblico e della moralità, all'assistenza morale e materiale dei cittadini», mentre la politica internazionale doveva assicurare «l'integrità, l'indipendenza e la dignità della Nazione Italiana, affidate ad efficienti e moderne Forze Armate, libere da ogni interferenza politica» ${ }^{10}$. La struttura del Fronte Nazionale consisteva in una sede centrale a Roma e una serie di delegazioni provinciali con sedi sparse in tutta Italia. Ai rappresentanti del Fronte in ciascuna provincia, selezionati da Borghese e dai suoi collaboratori, erano riservati i compiti di proselitismo e di studio di iniziative da assumere nel caso di lotta aperta ed armata contro i comunisti. A questo proposito, verso la fine del 1969, venne creata una struttura clandestina parallela a quella ufficiale, un "Gruppo B" occulto «destinato all'approntamento di "strumenti operativi" (essenzialmente: raccolta e conservazione armi, acquisizione di personale valido per azioni "disinvolte", approntamenti di "santuari") con caratterizzazione militare» ${ }^{11}$. Contemporaneamente il Comandante si

\footnotetext{
7 Nato ad Artena nel 1906 dalla principesca famiglia Borghese, nel corso della Seconda Guerra Mondiale fu ufficiale della Regia Marina e in seguito membro 'Decima Flottiglia Mas', unità speciale della Marina, della quale divenne Comandante. Dopo l'armistizio dell'8 settembre 1943 , decise di proseguire la guerra a fianco dei tedeschi e aderì alla Repubblica Sociale Italiana. Amnistiato nel dopoguerra dalla condanna per collaborazionismo, fu tra i fondatori del Movimento Sociale Italiano del quale fu nominato Presidente onorario nel 1951. Nel 1957 si allontanò dal partito in polemica con segreteria di Michelini, avvicinandosi alla destra extraparlamentare. Per approfondire si veda GREENE, Jack, MASSIGNANI, Alessandro, Il Principe nero. Junio Valerio Borghese e la X Mas, Milano, Mondadori, 2007; NESI, Sergio, Junio Valerio Borghese, un principe, un comandante, un italiano, Bologna, Lo Scarabeo, 2004. 8 Riservata raccomandata del Questore di Roma Melfi alla Direzione generale di Pubblica sicurezza, Divisione Affari Riservati del 1 ottobre 1968, prot. Rie. 224-25931, All. n. 235 in Allegati alla Relazione di perizia di GIANNULI, Aldo, del 12/03/97 per il Tribunale di Milano, procedimento penale n. 2/92F, p. 417 (d'ora in avanti GIANNULI 1997).

9 Fronte Nazionale, Orientamenti Programmatici, gennaio 1969, in PANSA, Giampaolo, Borghese mi ha detto, Milano, Palazzi, 1971, pp. 137-138.

${ }^{10}$ Ibidem.

${ }^{11}$ Appunto SID del 27 giugno 1974, n. 04/351/3, p. 1, All. n. 10 alla Relazione di perizia di GIANNULI, Aldo, n. 50 per la Procura della Repubblica di Brescia, procedimento penale $\mathrm{n}$. 91/97 mod. 21, p. 55 (d'ora in avanti GIANNULI n. 50).
} 
incontrò con i dirigenti delle formazioni di estrema destra come Ordine Nuovo e Avanguardia Nazionale per stabilire un patto di intesa che avrebbe implicato «la loro collaborazione alle attività organizzative e propagandistiche del Fronte» ${ }^{12}$.

Per lo sviluppo del suo movimento, Borghese sapeva di avere la necessità dell'appoggio delle Forze Armate e di finanziamenti da parte di gruppi industriali, pertanto sin dal 1969 aveva girato l'Italia e in particolare le città del Nord. A Genova, ad esempio, aveva incontrato i consensi di circa una quarantina di persone dell'ambiente economico-industriale, tra gli altri, l'industriale Guido Canale, l'armatore Alberto Cameli, l’imprenditore edile e presidente del Genoa F.C. Giacomo Berrino, l’industriale petrolifero Riccardo Garrone ${ }^{13}$.

Per il SID l'impostazione data dal Fronte veniva giudicata molto interessante e andava seguita con il massimo interesse, poiché essa rappresentava «il primo serio tentativo dell'estrema destra di un lavoro metodico di penetrazione e aggancio nell'ambiente militare, basato sull'ipotesi di una possibile "soluzione autoritaria" della crisi politica e sociale italiana» ${ }^{14}$.

Borghese, oltre agli ambienti economici e militari, si era assicurato di avere contatti anche con esponenti mafiosi ${ }^{15}$. Già nel corso del 1969, attraverso l'intermediazione di Felice Genoese Zerbi, fiduciario del Fronte a Reggio Calabria, il Principe era riuscito a mettersi in contatto con le 'ndrine calabresi, le quali a loro volta cercavano un interlocutore nelle forze dell'estrema destra. Il 26 ottobre 1969, il Comandante partecipò all'annuale summit mafioso presso la zona della Madonna di Polsi a Montalto per stringere un'alleanza con i capi 'ndranghetisti ${ }^{16}$. Anche Cosa Nostra era stata contattata dall'entourage del Principe Borghese. Un suo emissario aveva chiesto ai boss Pippo Calderone e Giuseppe Di Cristina se fossero stati disponibili a mettere a disposizione gli uomini delle cosche per un colpo di stato anticomunista in fase di avanzata preparazione. In cambio dei servigi resi sarebbe stato promesso l'alleggerimento della posizione processuale di alcuni importanti esponenti mafiosi

\footnotetext{
${ }^{12}$ Scheda riassuntiva sul Fronte Nazionale de1 22 marzo 1971, Archivio Servizio Informazioni Operative e Situazioni-Stato Maggiore aeronautica, p. 3, All. n. 236 in GIANNULI 1997, p. 421. ${ }_{13}$ Legione Territoriale Carabinieri di Genova, Appunto n.138802/1 del 18 giugno 1969, in Procura della Repubblica di Brescia, Procedimento penale n. 91/97 mod. 21, Documentazione acquisita Brescia, Fald. G/a-100, Fasc. 2, p. 157-159 (d'ora in avanti Brescia 91/97).

14 Ibidem.

15 FIGURELLI, Michele, Relazione della Commissione parlamentare d'inchiesta sul fenomeno della mafia e delle altre associazioni criminali similari, Relazione sullo stato della lotta alla criminalità organizzata in Calabria, 26 luglio 2000, pp. 94-95, in URL: <http://leg13.camera.it/_dati/leg13/lavori/doc/xxiii/o42/dooor.htm> [consultato il 30 agosto 2016].

${ }^{16}$ Ibidem.
} 
detenuti nonché la concessione, da parte del nuovo Governo, di un'amnistia ${ }^{17}$. Secondo la testimonianza di Antonino Calderone, il fratello Pippo e Giuseppe Di Cristina incontrarono Borghese a Roma per suggellare il patto. Il compito dei mafiosi sarebbe stato quello di affiancare le formazioni di destra che si sarebbero sostituite ai prefetti al momento stabilito ${ }^{18}$. Se l'operazione avesse avuto successo, in Sicilia sarebbe dovuta scoppiare un'insurrezione.

L'attività del Fronte si intensificò in maniera decisa nel corso dell'estate del 1970. I suoi uomini e quelli delle organizzazioni collegate si prepararono anche militarmente all'imminente azione eversiva, come ad esempio nei campi di "parasoccorso" allestiti da Sandro Saccucci, dirigente dell'Associazione Nazionale Paracadutisti d'Italia' ${ }^{19}$. Sia Ordine Nuovo che il neonato Movimento Politico Ordine Nuovo ${ }^{20}$ avevano inoltre mobilitato i propri aderenti per «campi di istruzione all'uso delle armi individuali e di reparto» e per lezioni avente come tema «Teoria della guerra rivoluzionaria, Organizzazione di un gruppo operativo rivoluzionario, Tecniche per il reperimento dei mezzi finanziari necessari all'azione politica di un movimento rivoluzionario» ${ }^{21}$.

\section{Gli Stati Uniti e il golpe}

Borghese non si preoccupava soltanto di avere l'appoggio dei gruppi di destra e di apparati militari italiani ma aveva cercato un contatto con gli ambienti dei servizi segreti statunitensi e con l'ambasciata americana a Roma. Si recò personalmente il 26

\footnotetext{
17 Interrogatorio di Tommaso Buscetta al G.I. di Palermo, 4 dicembre 1984; Deposizione di Tommaso Buscetta alla Commissione Parlamentare Antimafia, 16 novembre 1992; Interrogatorio di Antonino Calderone in sede di rogatoria internazionale a Marsiglia, 24 giugno 1987 e 9 novembre 1987, cit. in Tribunale Civile e Penale di Milano, Sentenza-ordinanza del G.I. SALVINI, Guido del 18/03/1995, p. 210 (d'ora in avanti SALVINI 1995).

${ }_{18}$ Audizione di Antonino Calderone alla Commissione Parlamentare Antimafia, 11 novembre 1992, cit. in BERTONI, Raffale, CIONI, Graziano, PARDINI, Alessandro, STANISCIA, Angelo, ATTILI, Antonio, BIELLI, Valter, CAPPELLA, Michele, GRIMALDI, Tullio, RUZZANTE, Piero, Stragi e terrorismo in Italia dal dopoguerra al 1974, Relazione della Commissione Stragi, Doc. XXIII n. 64, Volume I, tomo II, p. 300.

19 DE LUTIIS, Giuseppe, Relazione di perizia-Gladio ed eventuali collegamenti con la destra extraparlamentare, Tribunale di Bologna, Procedimento penale n. 1329/A/84, Tribunale di Bologna, Procedimento penale n. 1329/A/84, 1 luglio 1994, p. 168.

${ }^{20}$ Il Movimento Politico Ordine Nuovo era stato fondato nel dicembre 1969 da alcuni dissidenti ordinovisti, guidati da Clemente Graziani, i quali non condividevano la scelta di Pino Rauti (leader di ON) di rientrare nel MSI a seguito dell'elezione di Almirante a Segretario del partito. Vedi CONTI, Davide, op. cit., p. 66.

${ }^{21}$ Appunto SID proveniente dal Centro C.S. di Milano del 19 settembre 1970, p. 1, in Brescia 91/97, Faldoni "B/b" - atti AG Milano, Fald. B/b-1, Fasc. 7/3, p. 96; Dattiloscritto datato 3 giugno 1970, cit. in Tribunale di Roma, Sentenza nella causa penale di primo grado nei confronti di Clemente Graziani + 41 del 21/11/1973, pp. 119-121.
} 
gennaio 1970 all'ambasciata statunitense ma venne ricevuto solamente dal secondo segretario Charles Stout ${ }^{22}$.

L'accoglienza fu abbastanza fredda, pertanto Borghese e il suo entourage cercarono altre vie per ottenere almeno un silenzio-assenso all'iniziativa del colpo di stato da parte delle autorità di Washington. Il medico di Rieti Adriano Monti, appartenente ad Avanguardia Nazionale, secondo la sua testimonianza si mise in contatto, per conto del Principe, con Hugh Fenwich, ingegnere della Selenia e legale rappresentante del Partito Repubblicano statunitense in Italia. Costui si impegnò a parlare con Herbert Klein, l'assistente dell'allora Segretario di Stato Henry Kissinger, e di aggiornarlo in seguito sul colloquio.

Le opinioni di Klein si riassumevano in quattro punti: non dovevano essere impegnati civili e militari americani; all'operazione dovevano partecipare Carabinieri, Esercito, Marina, Aeronautica; si auspicava la costituzione di un Governo presieduto da un politico appartenente alla DC che godesse della fiducia degli USA; dovevano essere indette elezioni entro l'anno escludendo le liste comuniste ${ }^{23}$. Borghese, ascoltate le richieste di Klein, invitò Monti a ricontattare Fenwich affinché il governo americano indicasse una terna di nomi di gradimento per il futuro Presidente della Giunta militare. Il nome trasmesso fu uno solo: quello di Giulio Andreotti ${ }^{24}$.

Una conferma parziale del racconto di Monti, ma con significative divergenze, ci arriva dai documenti pubblicati dei Foreign relations of the United States. Nei primi giorni dell'agosto 1970 sia l'ambasciata che la centrale CIA di Roma vennero allertate a causa di un possibile tentativo di colpo di stato che avrebbe dovuto avere luogo la settimana del 10 agosto. L'ambasciatore Graham Martin inviò al Dipartimento di Stato un telegramma urgente da passare alla Casa Bianca ${ }^{25}$. Parlare di possibilità di colpo di stato in Italia era una cosa normale, spiegava l'ambasciatore, nel periodo di crisi di governo. Questo tentativo, tuttavia, non era così velleitario perché al contrario dei precedenti c'erano le prove di un coinvolgimento da parte di elementi politici e militari ${ }^{26}$. Un eminente uomo d'affari americano (Fenwich) era stato approcciato da

\footnotetext{
${ }^{22}$ Memorandum of conversation, The National Front and the Italian Political Situation, 26 gennaio 1970, URL:

<http://www.foia.cia.gov/sites/default/files/document_conversions/1705143/BORGHESE\%2 \%20JUNIO\%20VALERIO_0028.pdf> [consultato il 22 gennaio 2016].

${ }_{23}$ MONTI, Adriano, Il "Golpe Borghese". Un golpe virtuale allitaliana, Bologna, Lo Scarabeo, 2006, p. 55 .

24 Ibidem, p. 69.

${ }^{25}$ Telegram from the Embassy in Italy to the Department of State, Planning for possible coup attempt week of 10 August, August 7, 1970, doc. n. 196, in FRUS 1969-1976, vol. XLI Western Europe - NATO 1969-1972, Italy, p. 667 (d'ora in avanti Telegram August, 7, 1970).

${ }^{26}$ Ibidem.
} 
uno dei golpisti (Monti) il quale aveva affermato che il colpo di stato sarebbe stato supportato da elementi militari, della Confindustria e dei Carabinieri.

The American businessman was asked to ascertain whether the US would recognize such a regime if it came to power and whether he would see another member of the group [Remo Orlandini] for more details ${ }^{27}$. I asked him to do so [...]. He was told that:

(1) The organization involved is called Fronte Nazionale.

(2) The head of the organization is Valerio Junio Borghese.

(3) It is made up primarily of retired military personnel allegedly with broadly based contacts throughout Italian society (industrialists, labor unions and active military personnel), is not tied to any political party, is not monarchist in bent and has a para-military substructure.

(4) Its motivation is nationalistic, anti-Communist and pro-American.

(5) It has been meeting and organizing throughout Italy with the intent of having a coup d'état which will be assisted by the armed forces but once installed the military would be subordinated to civilian authority. Its state of readiness is such that it can be activated in six hours. Ten to fifteen days from now was estimated for effecting the coup. [...].

(7) They desire the U.S. Government to be informed and to recognize the group once they are installed in power. They ask no specific help and only request that their plans be kept secret and they not be exposed. [...].

(9) It was said specifically that operations would be mounted with or without US support ${ }^{28}$.

Martin si dimostrò contrario alla svolta autoritaria nel nostro Paese, ritenendo inoltre estremamente pericoloso il tentativo, anche se fosse fallito, poiché avrebbe potuto causare uno spostamento verso sinistra degli equilibri politici.

I am therefore conveying to Prince Borghese the message that we do not believe recourse to such measures are appropriate in present circumstances, and that our estimate is that, if attempted, they will not succeed. Therefore, he might be wise to emulate his collateral relative, Napoleon, and choose as his current code word the phrase "not tonight, Josephine"29.

27 Costruttore romano e numero due del Fronte.

${ }^{28}$ Telegram August, 7, 1970.

29 Ibidem. 
Il Segretario di Stato Rogers rispose al telegramma comunicando il suo scetticismo sull'effettiva possibilità di riuscita del colpo di stato e concordando con il diplomatico sul comportamento da tenere nei confronti di Borghese. Scrisse di aver allertato l'intelligence a Washington affinché fornisse all'ambasciatore informazioni non appena disponibili. Richiese inoltre il parere del diplomatico sull'opportunità di avvertire il presidente Saragat o il presidente del Consiglio Colombo ${ }^{30}$. Il giorno successivo Martin rispose al Segretario di Stato di aver avvisato il capo del SID Henke sulle manovre del Fronte, il quale aveva riferito al Ministro della Difesa Tanassi e a sua volta al Presidente Saragat ${ }^{31}$.

Martin, grazie all'aiuto del colonnello James Clavio, attaché militare dell'Ambasciata, continuò a seguire da vicino le trame golpiste. Nel mese di settembre il miliare americano avviò un'intensa attività informativa mirata ad acquisire informazioni sui gruppi della destra extraparlamentare, con la collaborazione di fonti introdotte nell'ambiente militare italiano. Dai suoi memorandum emerge come il Fronte fosse un'organizzazione da non sottovalutare dato che, se al momento non presentava le capacità di attuare un colpo di stato, avrebbe potuto acquisirle in un futuro non lontano32. Un pericolo che però il capo del SID Henke non sembrava aver preso in gran considerazione. Per una delle fonti contattate da Clavio, qualsiasi tentativo portato avanti da Borghese avrebbe avuto un effetto negativo sulle forze democratiche e avrebbe potuto suscitare l'effetto opposto di avvantaggiare i comunisti. Un'organizzazione come il Fronte, secondo la fonte, avrebbe dovuto essere controllata e utilizzata solamente quando le circostanze lo avrebbero permesso33. Il gruppo di Borghese poteva contare infatti su 5 mila attivisti e sui contatti con tutte le organizzazioni di destra. Costoro tuttavia diffidavano di Borghese in particolare per il suo legame con il servizio segreto inglese, dal quale il Comandante era, a loro dire, influenzato ${ }^{34}$. Anche in ambito militare Borghese aveva ottenuto scarsi consensi, tranne forse nell'ambiente della Marina. Per le fonti, il Principe aveva comunque ricevuto

${ }^{30}$ Telegram from the Department of State to the Embassy in Italy, Possible coup attempt, August 10, 1970, in CIPRIANI, Gianni, Relazione sui documenti concernenti l'italia rinvenuti negli archivi degli Stati Uniti, Commissione parlamentare d'inchiesta sul terrorismo in Italia e sulle cause della mancata individuazione dei responsabili delle stragi, 5 dicembre 2000, all. $\mathrm{n}$. 167.

${ }^{31}$ Telegram August, 7, 1970.

${ }^{2}$ Memorandum to the Ambassador from Army Attaché, Current Italian political situation, 1 September 1970, en URL:

<http://www.foia.cia.gov/sites/default/files/document_conversions/1705143/BORGHESE\%2C \%20 JUNIO\%20VALERIO_o031.pdf> [consultato il 22 gennaio 2016].

33 Ibidem.

34 Ibidem. 
finanziamenti da industriali e uomini d'affari come «COSTA, ex-President of Confindustria; FASSIO, a ship owner; MONTI, an oil man; and a DI FAINA»35.

I documenti statunitensi finora disponibili tuttavia non ci chiariscono le dinamiche dei mesi immediatamente precedenti al golpe, così come è evidentemente incongruente l'atteggiamento degli ambienti americani testimoniato da Monti, tutt'altro che restii ad appoggiare il golpe seppure a certe condizioni. Ciò che risulta chiaro, invece, è ancora una volta la dimostrazione che servizi di sicurezza, forze dell'ordine e finanche politici di spicco italiani, conoscevano l'esistenza e la pericolosità del piano di Borghese molti mesi prima della sua attuazione.

\section{Il racconto del golpe}

L'opera di penetrazione attuata dagli esponenti del Fronte si era intensificata nel corso dei mesi antecedenti al golpe soprattutto nei confronti delle Forze Armate. Gli aderenti al movimento di Borghese ritenevano che grazie all'accensione di focolai di disordine o di episodi di violenza che ponessero in evidenza la pericolosità per le istituzioni, si sarebbe potuta determinare una reazione da parte di quelle forze «a cui st[esse] ancora a cuore la salvaguardia dei principi d'ordine»36. Costoro avrebbero dovuto pertanto prendere in mano la situazione all'insorgere dei primi disordini. «Tutti i "frontisti" sapranno far loro comprendere di essere ampiamente disponibili per una attività "fiancheggiatrice", nonché pronti, come civili, ad assumere ogni opportuna responsabilità nei posti-chiave riguardanti la vita del Paese»37.

Nei giorni antecedenti alla cosiddetta "notte di Tora Tora" fervevano i preparativi $3^{3}$. In particolare a Montesacro Alto, dove il costruttore Remo Orlandini, braccio destro del Principe, aveva i suoi uffici e cantieri di lavoro, si intensificavano le visite dei delegati provinciali del Fronte Nazionale e le riunioni per definire gli ultimi dettagli del piano. Gli obiettivi dell'azione, che si sarebbe svolta principalmente a Roma, erano il Ministero degli Interni e quello della Difesa, «punti chiave per l'ulteriore sviluppo dell'azione insurrezionale»39. Contemporaneamente erano previste le occupazioni della sede della RAI-TV, delle centrali elettriche e telefoniche, nonché l'accensione di gravi

\footnotetext{
35 Ibidem.

${ }^{36}$ Tribunale penale di Roma, Sentenza-ordinanza del G.I. FIORE, Filippo n.3361/71 R.G.P.M., n.1054/71 R.G.G.I., 5 novembre 1975, p. 75 (d'ora in avanti FIORE 1975).

37 FIORE 1975, p. 75.

${ }^{38}$ L'operazione golpista venne chiamata in questo modo dal nome in codice utilizzato dai giapponesi nell'attacco a sorpresa contro gli Stati Uniti a Pearl Harbor, avvenuto proprio la notte tra il 7 e l'8 dicembre del 1941.

39 FIORE 1975, p. 80.
} 
disordini in vari punti della città, «al fine di determinare il decisivo e tanto atteso intervento dei militari»40. Il proclama che Borghese avrebbe dovuto rivolgere alla nazione era pronto così come il programma operativo del nuovo regime. Tra i punti principali figuravano: il mantenimento dell'Italia all'interno dell'Alleanza atlantica, la nomina di un inviato speciale negli Stati Uniti per discutere di una eventuale partecipazione italiana nel conflitto del Vietnam e la richiesta di un prestito per fronteggiare la crisi economica ${ }^{41}$.

Secondo le decisioni del Comandante Borghese, Avanguardia Nazionale avrebbe dovuto avere due propositi: alcuni commandos avevano il compito di far saltare in aria tutte le strade che avrebbero potuto permettere alle unità dell'esercito di stanza ad Anzio-Nettuno (secondo il Fronte fedeli al presidente Saragat) di raggiungere Roma, mentre la maggior parte dei membri della formazione di estrema destra avrebbe dovuto occupare il Ministero degli Esteri42. All'alba del giorno successivo avrebbero dovuto essere rimpiazzati da truppe regolari e destinati ad un altro incarico di fiducia: rastrellare, con l'aiuto dei Carabinieri, una serie di persone che veniva ritenuto «opportuno allontanare coattivamente da Roma per qualche tempo» per mezzo di alcune navi pronte nel porto di Civitavecchia43. Il fatto di essere subito estromessi da un punto-chiave quale il Ministero degli Interni e naturalmente il timore che tutto il golpe potesse rivelarsi una trappola per stroncare l'estrema destra provocò non poche perplessità e proteste. Il numero uno di AN, Stefano Delle Chiaie con l'appoggio del dott. Salvatore Drago, medico della Polizia, riuscì ad ottenere un incarico differente ${ }^{44}$. Il 6 dicembre venne così deciso che Avanguardia avrebbe avuto il compito di occupare il Ministero degli Interni. Anche al di fuori di Roma i membri dell'apparato erano pronti ad intervenire, come ad esempio a Venezia, Verona, Reggio Calabria ma anche in località certamente non strategiche come Passignano, presso il lago Trasimeno ${ }^{45}$. Una

40 Ibidem, p. 82.

${ }^{41}$ Ibidem, pp. 81-83.

42 Relazione riservata su Avanguardia Nazionale e gruppi collegati, senza data [novembre 1972], Documentazione sequestrata presso la sede di "OP", in COMMISSIONE PARLAMENTARE D'INCHIESTA SULLA LOGGIA MASSONICA P2, Allegati alla relazione, Serie II: Documentazione raccolta dalla Commissione, Vol. III, Tomo IV, Parte I, p. 760-769 (d'ora in avanti COMMISSIONE P2).

43 Ibidem.

44 Per le dichiarazioni di Orlandini su Drago, FIORE 1975, p. 85. Drago è una figura particolare perché oltre ad essere legato ad AN era anche in stretti rapporti con il direttore dell'Ufficio Affari Riservati del Ministero dell'Interno Federico Umberto D'Amato.

45 Vedi le testimonianze di Carlo Digilio, Martino Siciliano, Enzo Ferro, Vincenzo Vinciguerra, Andrea Brogi. Interrogatorio di Carlo Digilio del G.I. Salvini, 6 aprile 1994, in Brescia 91/97, Verbali Digilio, Fald. D/c-1, p. 62; deposizione di Enzo Ferro al G.I. Salvini, 1 luglio 1992, cit. in SALVINI 1995, p. 119; deposizione di Vincenzo Vinciguerra, 13 gennaio 1992, cit. in ivi, p. 238; 
nota della Questura di Roma datata 17 novembre 1970 fornisce un quadro completo dell'organizzazione del Fronte a qualche settimana dal golpe:

[...] il Fronte Nazionale, da circa tre mesi starebbe preparando un piano insurrezionale su scala nazionale. [...]. Il maggiore ROSA Mario [...] avrebbe già approntato dischi e fasce tricolori per distribuirle ai capi gruppi per il riconoscimento. Si è appreso [...] che a seguito di una riunione cui avrebbero partecipato una ventina di elementi di estrema destra, [...] sono stati fatti anche riferimenti ad altre personalità che sarebbero coinvolte con il colpo di stato fra cui l'Ammiraglio di Squadra Navale M.O.V. Gino BIRINDELLI che il 18.10.1970, ha cessato dalla carica di comandante della squadra navale italiana ed è passato alla Nato. Il maggiore Rosa sarebbe anche in contatto con il generale di divisione dello Stato Maggiore Esercito RADICE PANNARIA e con il colonnello BONINO Giovanni dell'Ufficio smistamento truppe, compartimento Stazione Termini. Altri contatti sono stati citati a seguito delle riunioni che sarebbero in corso con un colonnello dell'Aeronautica militare e con un direttore dei servizi tecnici della R.A.I. T.V. per un eventuale proclama al popolo italiano della instaurazione di un governo militare e di tecnici. Sembra che tali personalità abbiano preso già contatti con elementi della C.I.A. U.S.A., i quali avrebbero conferito il loro appoggio ma solo successivamente al colpo di stato ${ }^{46}$.

Il 7 dicembre 1970 l'azione era pronta. Nel corso della serata, i responsabili 'politici', le menti dell'operazione eversiva, si riunirono nell'ufficio di Mario Rosa in via S. Angela Merici. Il gruppo, capeggiato dallo stesso Borghese, era composto oltre al Rosa, dal generale dell'Aeronautica a riposo Giuseppe Casero, dal colonnello dell'Aeronautica Giuseppe Lo Vecchio e dal capitano dei Carabinieri Salvatore Pecorella47. Da questa sede il Principe manteneva i contatti con i diversi gruppi operativi e con le persone che avrebbero dovuto intervenire in appoggio esterno ai congiurati. A Remo Orlandini spettava il compito di dirigere e coordinare i modi ed i tempi di intervento dei gruppi dislocati nelle diverse zone della città. Il "comando operativo" dell'azione eversiva, era pertanto riunito nel cantiere del costruttore romano. L'occupazione del Ministero dell'Interno era l'obiettivo principale dei golpisti. Già nel primo pomeriggio del 7 dicembre, un commando di Avanguardia Nazionale guidato da Delle Chiaie, era penetrato nell'armeria del Viminale con la complicità del

deposizione di Andrea Brogi al G.I. Salvini, 9 gennaio 1992, in Brescia 91/97, Verbali Milano, Fald. D/b-2, p. 583 .

${ }^{46}$ Appunto Questura di Roma, Fronte Nazionale, 17 novembre 1970, All. n. 9 in GIANNULI n. 50, pp. 49-50.

47 FIORE 1975, p. 86. 
Capitano Enzo Capanna, aiutante maggiore del Capo del Reparto Autonomo Guardie di Pubblica Sicurezza. Gli avanguardisti si adoperarono per predisporre all'impiego i circa duecento mitra custoditi nell'armeria. Verso sera anche il gruppo di Rieti guidato da Adriano Monti raggiunse il Viminale per velocizzare le operazioni e per predisporre la seconda fase dell'azione, l'occupazione della centrale radiotelefonica del Ministero. Verso mezzanotte il camion su cui vennero caricate le armi era pronto per partire e ad attenderlo non c'era solamente il gruppo di Orlandini ma anche i paracadutisti capeggiati da Sandro Saccucci, riuniti nella palestra di via Eleniana, sede dell'associazione ${ }^{48}$. Contemporaneamente un gruppo di mafiosi avrebbe dovuto tenere d'occhio il capo della Polizia Vicari per poterlo eliminare non appena avessero ricevuto l'ordine ${ }^{49}$. Un altro commando capeggiato dal futuro Gran Maestro della Loggia P2 Licio Gelli, avrebbe invece dovuto catturare il Presidente della Repubblica Saragat50.

Il piano dei congiurati sembrava pertanto poter procedere senza intoppi. Dalla Scuola della Guardia Forestale di Cittaducale, in provincia di Rieti, partì un'autocolonna composta da 197 uomini, guidati dal direttore, il colonnello Luciano Berti e armati di tutto punto e diretta presumibilmente verso gli studi Rai di via Teulada51. Poco prima della mezzanotte, nel frattempo, Sandro Saccucci lasciò la palestra di via Eleniana per dirigersi verso il cantiere di Orlandini promettendo di tornare al più presto con le disposizione operative, le quali molto probabilmente, sarebbero state quelle di arrestare parlamentari e uomini politici52. Nello stesso cantiere di Montesacro, Lo Vecchio e Casero stavano aspettando il momento opportuno per prelevare il Capo di Stato Maggiore dell'Aeronautica, generale Duilio Fanali, grazie al cui appoggio i congiurati avrebbero occupato il Ministero della Difesa53. Un'altra persona era presente al "comando operativo" dell'azione golpista. Si trattava di Hugh Fenwich, il già citato rappresentante del Partito Repubblicano statunitense, il cui compito sarebbe stato quello di contattare il Presidente Nixon con una telefonata che avrebbe dovuto passare sia attraverso la base NATO di Napoli, che il comando navale NATO di stanza nell'isola di Malta54. In questo momento iniziarono i primi problemi

\footnotetext{
48 FIORE 1975, p. 97.

49 Trascrizione della conversazione del 31 maggio 1974 tra Sandro Romagnoli, Maurizio Degli Innocenti, Torquato Nicoli e Antonio Labruna, cit. in SALVINI 1995, pp. 207-209.

50 Ibidem, pp. 202-205.

${ }^{51}$ I forestali disponevano di pistole d'ordinanza Beretta 7.65; moschetti mod. 91/38; 53 mitra M.A.B., 1300 cartucce per calibro 7.65, 1728 per moschetto, 4700 per M.A.B. e uno strumento a getto utilizzabile come lanciafiamme. Vedi FIORE 1975, p. 105.

$5^{2}$ Ibidem, p. 101; FLAMINI, Gianni, Il partito del golpe. Le strategie della tensione e del terrore dal primo centrosinistra organico al sequestro Moro, 1968-1970, Ferrara, Bovolenta, 1982, p. 225.

53 FLAMINI, Gianni, op. cit., p. 223.

54 Ibidem, p. 220.
} 
per i golpisti, poiché la prevista telefonata di Fenwich si sarebbe "arenata" nella base NATO della città partenopea. Poco dopo l'una di notte, Borghese, in attesa frenetica di dare il via definitivo al golpe, ricevette una telefonata. Al termine di un breve scambio di battute, il Principe si rivolse ai suoi compagni informandoli che l'operazione era stata annullata, a causa della mancata «collaborazione di un "gruppo di ufficiali" che avrebbero dovuto aprire dal di dentro, il portone del Ministero della Difesa»55. Alle richieste di spiegazione, Borghese si mostrò reticente, limitandosi a dichiarare di aver «obbedito ad ordini superiori»56. Oramai l'importante, pertanto, era fermare l'operazione e senza che ci fossero conseguenze spiacevoli. L'autocolonna di Berti venne bloccata a poche centinaia di metri dalla sede della RAI, mentre più difficile fu rintracciare il camion con le armi che era partito dal Viminale e farlo rientrare. I mitra vennero ricollocati al loro posto, tuttavia un esponente di Avanguardia Nazionale riuscì a trafugare una pistola mitragliatrice Beretta. Almeno per il momento, tuttavia, sembrò che nessuno si fosse accorto di nulla, evitando in questo modo gravi conseguenze per i congiurati delusi e arrabbiati.

\section{Indagini e nuove trame}

Nonostante l'insuccesso dell'iniziativa, gli esponenti del Fronte Nazionale dovettero constatare di essere riusciti a mantenere il segreto sulla parte del programma già realizzato e sugli eventi della notte di Tora Tora. I partecipanti al tentativo di colpo di stato si poterono pertanto riunire indisturbati nella sede del Fronte il 17 gennaio 1971 dove Borghese, duramente criticato, venne estromesso dall'organizzazione 57 . Nei due incontri successivi tra gli esponenti del Fronte, ai quali significativamente Borghese non prese parte, si cercò di ristrutturare il movimento e di rimettere in moto l'azione eversiva. La fase di ristrutturazione dell'organizzazione golpista tuttavia durò poco. Il quotidiano di Roma vicino al Partito Comunista, «Paese Sera», il 17 marzo 1971 pubblicò in prima pagina la notizia dell'avvenuto tentativo di colpo di stato da parte del principe Borghese e degli arresti disposti dalla procura della Repubblica di Roma nei confronti dello "stato maggiore" del Fronte. Borghese tuttavia riuscì ad evitare la cattura rifugiandosi in Spagna.

Sin dai primi giorni di dicembre del 1970, gli uomini del SID e dell'Ufficio Affari Riservati avevano infatti provveduto a tenere sotto stretto controllo i cospiratori poiché,

57 Ibidem, p. 137. 
già nel corso della notte tra il 7 e l'8 dicembre, erano stati allertati in merito ad una azione imprecisata nei confronti del Viminale condotta dagli uomini del Fronte. Una nota inviata all'Ufficio D di Roma (la sezione dedicata al controspionaggio) descrive l'attività del Fronte post-golpista «tale da potere rappresentare un motivo di turbamento nell'attuale, delicata situazione contraddistinta da difficili equilibri politici, nonché da spinte sovversive delle più svariate provenienze»58. La stessa Questura romana teneva sotto osservazione gli uomini Borghese. È datata 28 gennaio infatti un'informativa particolareggiata sul Fronte e sulla sua attività di propaganda «allo scopo di rinvigorire le proprie file e stabilire le premesse necessarie per l'attuazione di un nuovo piano politico antigovernativo e prettamente rivoluzionario»59. La fonte interrogata dalla Questura confermava inoltre che qualcosa di pericoloso per lo Stato fosse effettivamente avvenuto nella notte tra il 7 e l'8 dicembre ${ }^{60}$. Secondo Antonino Di Spirito, esponente deluso del Fronte Nazionale, «affluirono a Roma circa 300 persone, armate di mitra e pistole, poiché doveva scattare un dispositivo di emergenza. Mentre alcuni reparti militari avrebbero dovuto porre in atto il colpo di stato, esse avrebbero dovuto neutralizzare i punti strategici della Capitale, compresa la Questura». Il mancato intervento dei militari, che, inspiegabilmente, quella notte non si fecero vivi, avrebbe pertanto fatto saltare il golpe ${ }^{61}$.

È interessante notare che Di Spirito pensasse ad un colpo di stato attuato non grazie all'aiuto dei militari bensì messo in atto dai militari stessi e con l'aiuto, subordinato, dei civili. Descritto in questi termini il tentativo di Borghese assume nuova luce. Non più, quindi, un velleitario tentativo portato avanti da gruppi di esaltati appartenenti all'estrema destra che vantava qualche appoggio nei reparti delle Forze Armate, bensì un serio pericolo per la sicurezza dello Stato democratico. Un altro appunto della Questura romana, inoltre, riportava che il Fronte Nazionale era «inserito in un gioco di industriali, CIA, PSU, militari, al fine di favorire non tanto un colpo di stato ma "un colpo d'ordine", condotto dal PSU e da altri gruppi di potere che si trovano dietro questo partito» ${ }^{62}$. Esso infatti era «favorevole ad abdicare ad un colpo di stato fascista, per un colpo di stato d'ordine» tale da consolidare gli assetti del potere costituito e non quindi finalizzato a scardinarli63. Un'altra nota proveniente dalla Questura di Roma del

${ }^{8}$ Appunto SID, Attività del Fronte Nazionale, 16 gennaio 1971, Allegato n. 5 in GIANNULI n. 50, pp. 32-35.

${ }_{59}$ Nota della Questura di Roma, 28 gennaio 1971, Allegato n. 6, in ibidem, pp. 37-39.

6o Ibidem.

${ }^{61}$ Relazione del Brg. M. Lombardi alla Questura di Roma, 27 gennaio 1971, Allegato n. 8, in ivi, pp. 45-47.

${ }^{62}$ Nota della Questura di Roma, 23 febbraio 1971, Allegato n. 4, in GIANNULI n. 50, p. 29.

63 Ibidem. 
7 marzo avvalora questa interpretazione poiché riportava che all'«azione antigovernativa e rivoluzionaria» programmata dal Fronte Nazionale non sarebbero stati interessati solamente i movimenti di estrema destra «ma soprattutto gli ambienti legati alla socialdemocrazia, ai repubblicani, ai democristiani, ai liberali, nonché alla massoneria romana e fiorentina. In particolare vengono fatti i nomi del Presidente dalla Repubblica, degli Onn.li Leone, Pella, Preti, Tanassi, Piccoli»64. L'operazione avrebbe dovuto prevedere «una stretta collaborazione di alcuni reparti dell'Arma dei Carabinieri, della Guardia di Finanza, dell'esercito e del Compartimento romano della Polizia Stradale»65.

Si può ipotizzare pertanto un golpe "a doppio fondo" il cui scopo palese (la conquista del potere da parte di un gruppo eversivo) mascherava un gioco di potere. Abbiamo dunque molteplici indicazioni che convergono nell'indicare il golpe Borghese come un'azione di stabilizzazione del sistema di potere basata sull'intesa con significative correnti dei partiti di centro. Ad avvalorare ulteriormente questa interpretazione ci giungono in soccorso le testimonianze di due personaggi legati all'estrema destra. Il primo Paolo Aleandri, neofascista, era venuto in contatto con l'ordinovista Fabio De Felice. In relazione al golpe, De Felice spiegò ad Aleandri la «geografia dei gruppi», $\mathrm{i}$ «tre livelli» che avrebbero dovuto partecipare: «un livello che era costituito da gruppi estremisti della destra eversiva, una parte che era costituita da persone che avevano in mente una svolta autoritaria» e infine «una terza parte costituita dagli ambienti che in forma più o meno palese venivano contattati, però non con l'esplicita richiesta di aderire a un golpe, quanto avvicinandoli a certe posizioni che implicassero un loro consenso [...] per una democrazia forte»66. Sempre grazie ai racconti di De Felice, Aleandri era venuto a conoscenza del fatto che la parte centrale del golpe Borghese, dovesse «essere congegnata in modo da avere l'ausilio di ufficiali dei carabinieri» i quali avrebbero dovuto dare «un segnale che consentisse poi, a tutti i comandi d'Italia di trarre dalle casseforti [...] dei piani antinsurrezionali» ${ }^{67}$. Il golpe, pertanto, avrebbe avuto la partecipazione di pochi elementi consapevoli e la partecipazione inconsapevole dei comandi, i quali avrebbero obbedito a degli ordini

\footnotetext{
${ }^{64}$ Nota della Questura di Roma, 23 febbraio 1971, Allegato n. 17, p. 1-2, in ibidem, pp. 102-103. 65 Ibidem.

66 Audizione di Paolo Aleandri alla Commissione P2, 9 febbraio 1984, COMMISSIONE P2, Allegati alla relazione, Serie II: Documentazione raccolta dalla Commissione, vol. III, t. IV, Parte I, p. 88.

67 Audizione di Paolo Aleandri alla Commissione P2, 9 febbraio 1984, COMMISSIONE P2, Allegati alla relazione, Serie II: Documentazione raccolta dalla Commissione, vol. III, t. IV, Parte I, p. 86 .
} 
prestampati per occasioni insurrezionali ${ }^{68}$. Il secondo personaggio è invece Amos Spiazzi, militare veronese legato a Ordine Nuovo e protagonista del successivo tentativo eversivo del gruppo denominato Rosa dei Venti. Nel corso della sua audizione alla Commissione $\mathrm{P} 2$, raccontò che la sera del 7 dicembre ricevette l'ordine di attuare l'«esigenza triangolo», ovvero «l'impiego immediato effettivo, di tutto quell'apparato anticomunista [...] che comprendeva appunto ufficiali, sottufficiali e soldati di sicura fede», e dirigersi verso Sesto San Giovanni, considerata una "zona calda"69. A metà strada, tuttavia era arrivata la comunicazione che si trattava soltanto di un'esercitazione. Secondo Spiazzi, l'ordine di bloccare sia il golpe Borghese che l' "esigenza triangolo" era arrivato da Andreotti7o.

All'indomani dello scoop di «Paese Sera», Gasca Queirazza, capo del Reparto "D" del SID, preparò un rapporto a Miceli sull'attività del Fronte Nazionale. Il SID, ancora una volta, propendette per la scarsa pericolosità del Fronte il quale non sembrava potesse «costituire una minaccia alla sicurezza interna»71. Inoltre, per quanto riguardava le «iniziative eversive, il Fronte [era] stato fino ad oggi inesistente»72. In merito alla notte del 7 dicembre, l'iniziativa, dagli accertamenti svolti dal SID, «sarebbe stata interrotta a causa dei contrasti, tentennamenti e ripensamenti emersi tra i fautori» 73 . Non risultava, inoltre, che questa «mirasse ad un colpo di stato», tuttavia la situazione veniva «attentamente seguita»74. Nonostante il rapporto non fosse certamente compromettente per il Fronte, il generale Miceli rifiutò la proposta del capo dell'Ufficio "D" di inoltrare l'appunto a tutte le autorità interessate al caso75. La Questura di Roma invece si premurò di inviare all'autorità giudiziaria tutti gli elementi riscontrati nelle indagini svolte ${ }^{76}$. Il 2 marzo il Questore, inoltre, inviava una lettera al Ministro dell'Interno contenente notizie dettagliate sulle perquisizioni e sul materiale sequestrato agli uomini del Fronte, in particolare «un'agenda, contenente nomi di esponenti della destra politica italiana, di ufficiali delle Forze Armate, in servizio

68 Ibidem, p. 88.

69 Audizione di Amos Spiazzi alla Commissione P2, 25 novembre 1983, in COMMISSIONE P2, Allegati alla relazione, Serie II: Documentazione raccolta dalla Commissione, vol. III, t. XI, pp. 475-530.

70 Ibidem.

${ }^{71}$ Rapporto SID, Attività del Fronte Nazionale, 18 marzo 1971, p. 5, in COMMISSIONE P2, Allegati alla relazione, Serie II: Documentazione raccolta dalla Commissione, vol. III, t. IV, Parte I, p. 183.

72 Ibidem.

73 Ibidem, p. 179.

74 Ibidem, p. 180.

75 FIORE 1975, p. 162.

${ }^{76}$ Lettera del Vice Questore di Roma Provenza alla Procura della Repubblica di Roma, 18 marzo 1971, pp. 2-4, Allegato n. 243 in GIANNULI 1997, pp. 457-459. 
permanente ed in congedo»77. Anche al SID, tuttavia, si proseguivano le indagini nonostante non si ritenesse particolarmente significativa l'azione di Borghese. Un centro periferico del servizio italiano in un report, oltre a confermare l'ingresso dei giovani di Avanguardia all'interno del Viminale (addirittura convocati dal vice di Federico Umberto d'Amato dell'Ufficio Affari Riservati), segnalava che sarebbe stato il ministro Restivo a fornire a «Paese Sera» la notizia del fallito golpe, il quale avrebbe commentato «Per ora abbiamo salvato la Repubblica»78. Tra le personalità non all'oscuro degli avvenimenti sarebbero state, secondo la fonte, «l'Amm. Birindelli, in funzione del suo incarico, il Capo di S.M. della Marina, il Capo di S.M. dell'Esercito, il Comandante della III Armata, il Comandante delle Fanterie del Sud-Europa e alcune personalità del Quirinale»79.

Grazie alla documentazione ricevuta dalla Questura, la Procura di Roma diede il via alle indagini. Tuttavia, i magistrati ritennero che le notizie raccolte non fossero sufficienti affinché l'inchiesta potesse procedere. Il Giudice Istruttore, pertanto, inviò una lettera nel luglio 1971 al direttore del SID chiedendo che il servizio rendesse disponibile all'autorità giudiziaria ogni risultanza acquisita. Il generale Miceli rispose alla missiva il 13 agosto specificando che «ogni ricerca informativa in merito svolta dal Servizio, nel quadro dei compiti istituzionali, ha portato all'esclusione di collusioni, connivenze o partecipazioni di ambienti o persone militari in attività di servizio» ${ }^{80}$. I magistrati ritennero di non avere abbastanza elementi per chiedere il rinvio a giudizio degli arrestati e l'inchiesta pertanto subì una brusca interruzione. Nel febbraio del 1972 gli eversori del Fronte vennero scarcerati e per Borghese venne revocato il mandato di cattura.

Dopo il cambio al vertice dell'Ufficio D del SID nel 1972, anche il servizio italiano decise di seguire da vicino i gruppi eversivi. L'azione informativa, affidata da Gianadelio Maletti al capitano Antonio Labruna, diede presto i suoi frutti ${ }^{81}$. Nei primi

\footnotetext{
77 Riservatissima del Questore di Roma Parlato alla Direzione generale di Ps, Divisione Aarr del 2 aprile 1971, p. 4, Allegato n. 244; Ibidem, p. 466.

${ }^{78}$ Figura interessante è anche quella di Federico Umberto D'Amato, direttore dal 1971 al 1974 dell'Ufficio Affari Riservati, il servizio segreto civile gestito dal Ministero dell'Interno in stretto contatto con la CIA. Per approfondire vedi PACINI, Giacomo, Il cuore occulto del potere: storia dell'Ufficio Affari Riservati del Viminale (1919-1984), Roma, Nutrimenti, 2010.

79 Rapporto SID, Italia-Situazione interna, 2 aprile 1971, p. 1-2, in COMMISSIONE P2, Allegati alla relazione, Serie II: Documentazione raccolta dalla Commissione, vol. III, t. IV, Parte I, pp. 187-188.

80 FIORE 1975, pp. 164-165.

${ }^{81}$ Sia Maletti che Labruna saranno in seguito tra i protagonisti del processo per la strage di Piazza Fontana in quanto accusati di aver cercato di far evadere Giovanni Ventura e favoreggiamento personale nei confronti di Guido Giannettini e Marco Pozzan. Verranno condannati rispettivamente a 4 e 2 anni di reclusione. Entrambi i loro nomi, inoltre, risultano
} 
mesi del 1973 un armatore napoletano rivelò a Labruna di essere molto preoccupato dal fatto che potessero nuovamente verificarsi «iniziative golpiste analoghe a quelle del 1970 cui egli stesso aveva partecipato mettendo le proprie navi a disposizione per il trasporto degli internati» ${ }^{82}$. L'imprenditore gli disse di poterlo mettere in contatto con Remo Orlandini, poiché costui «continuava ad agitarsi»83. Si trattava infatti del tentativo eversivo del gruppo la Rosa dei Venti, composto da elementi del Fronte, ad esclusione di Borghese, assieme a militanti di destra e militari del Nord Italia. Labruna iniziò a incontrarsi frequentemente con il costruttore romano presentandosi come capitano dei carabinieri del SID, fingendo di voler aderire alla causa eversiva per carpire tutti i particolari utili sulla struttura dell'organizzazione, i nomi dei membri, i contatti con militari e i loro nomi. Via via Orlandini iniziò a fidarsi del suo interlocutore e progressivamente cominciò a rivelare informazioni interessanti, anche sul tentativo precedente del dicembre 1970, come, ad esempio, sull'appoggio che il capo del SID Miceli avrebbe dato all'operazione e i suoi incontri con Borghese ${ }^{84}$. Anche la magistratura tuttavia aveva iniziato ad indagare sulle nuove trame, pertanto Orlandini dovette fuggire a Lugano per non essere arrestato. Qui proseguirono i suoi incontri con Labruna ed altri esponenti del Fronte (Tino Nicoli e Maurizio Degli Innocenti) che nel frattempo il capitano del SID aveva convinto a collaborare assieme al suo superiore, il colonnello Sandro Romagnoli. Le registrazioni degli incontri e le rivelazioni che Orlandini fece a proposito della notte di Tora Tora furono il piatto forte del dossier che il Servizio preparò qualche mese più tardi ${ }^{85}$. Il rapporto, che includeva i risultati dell'attività investigativa degli ultimi due anni nei confronti dei gruppi eversivi di destra, dopo varie consultazioni tra il Ministro della Difesa Andreotti e i vertici delle Forze Armate e dell'Ordine (compreso il nuovo capo del SID Casardi), pervenne ai

nell'elenco degli appartenenti alla Loggia P2. Sulla Loggia P2 vedi FLAMIGNI, Sergio, Trame atlantiche. Storia della Loggia massonica segreta P2, Milano, Kaos, 2005.

${ }^{82}$ Deposizione resa dal capitano Labruna al G.I. Salvini, 9 ottobre 1992, p. 3 in Brescia 91/97, Testi sentiti e prodotti, Labruna Antonio, p. 214.

83 Deposizione resa dal capitano Labruna al G.I. Tamburino, 31 ottobre 1974, p. 1, in COMMISSIONE P2, Allegati alla relazione, Serie II: documentazione raccolta dalla Commissione, vol. III, t. IV, Parte I, p. 597.

84 Trascrizione della conversazione del 28 giugno 1973 tra Antonio Labruna e Remo Orlandini, p. 1-2, in COMMISSIONE P2, Allegati alla relazione, Serie II: documentazione raccolta dalla Commissione, vol. III, t. IV, Parte I, pp. 282-283.

85 Trascrizione della conversazione del 17 giugno 1974 tra Antonio Labruna, Sandro Romagnoli, Remo Orlandini, Torquato Nicoli e Maurizio Degli Innocenti, in Brescia 91/97, Documentazione trasmessa dal G.I. di Milano, Fald. G/a-143, Fasc. 3, pp. 2-24. 
magistrati romani il 3 novembre $1974^{86}$. Nel frattempo, a Cadice, il 26 agosto, Borghese era morto improvvisamente, «con felice coincidenza», all'età di 68 anni ${ }^{87}$.

Il 5 novembre 1975 il giudice istruttore Filippo Fiore rinviava a giudizio 78 persone per il tentato golpe Borghese e per i tentativi eversivi successivi ${ }^{88}$. Per costruire l'impianto accusatorio erano state di notevole aiuto le registrazioni effettuate da Labruna contenenti le rivelazioni di Orlandini. Una prova fondamentale della veridicità delle sue affermazioni venne trovata dai magistrati nel momento in cui fu ispezionata l'armeria del Viminale per constatare se corrispondesse al vero ciò che il costruttore romano aveva detto all'agente del SID in merito alla sostituzione della pistolamitragliatrice trafugata ${ }^{89}$. Nomi importanti citati nelle informative dei servizi e della Questura come legati ai tentativi eversivi vennero tuttavia prosciolti (Miceli, Drago) o archiviati dallo stesso giudice istruttore (Fanali, Fenwich) ${ }^{90}$. Nonostante il Fronte venisse considerato un'associazione «fondata e organizzata per arrivare ad un sovvertimento radicale dei poteri pubblici mediante strumenti rivoluzionari», a molti appartenenti venne risparmiato il processo poiché, per il giudice, «molte persone aderirono al Fronte nazionale perché illuse o confuse da una ingannevole pubblicità»91. Il 14 luglio 1978 la Corte d'Assise, al termine del giudizio di primo grado, affermò l'insussistenza del delitto di insurrezione armata per i fatti del 7-8 dicembre 1970. Degli iniziali 78 imputati, soltanto 46 vennero condannati. Ancora più benevola la sentenza della Corte d'Appello, confermata poi in Cassazione, la quale assolse tutti gli imputati, anche i rei confessi, "perché il fatto non sussiste». Per i giudici romani «i "clamorosi" eventi della notte in argomento» non erano nient'altro che il «conciliabolo di quattro o cinque sessantenni» 92 .

\footnotetext{
86 Appunto SID del 27 giugno 1974, n. 04/351/3, p. 1, Brescia 91/97, Annotazioni di Polizia Giudiziaria, ROS Roma, Fald. C/a-01, Fasc.4, p. 689.

${ }^{87}$ L'espressione è del generale Viviani, uno dei successori di Maletti all'Ufficio D del SID, vedi VIVIANI, Ambrogio, Storia dei servizi segreti italiani, Roma, ADN-Kronos Edizioni, 1986, p. 175 .

88 NUNZIATA, Claudio, «Golpe Borghese» $e$ «Rosa dei Venti». Come si svuota un processo, in BORRACCETTI, Vittorio (a cura di), Eversione di destra, terrorismo, stragi, Milano, Franco Angeli, 1986, p. 71.

89 Orlandini aveva confessato a Labruna di essersi adoperato per procurare una copia sostitutiva della pistola-mitragliatrice trafugata dagli esponenti di AN. I magistrati, con l'aiuto dei tecnici della Beretta, constatarono, in effetti, che i numeri di serie di un arma presente all'interno del Viminale non corrispondevano e che la stessa risultava contraffatta. Vedi Trascrizione della conversazione del 17 giugno 1974 tra Antonio Labruna, Sandro Romagnoli, Remo Orlandini, Torquato Nicoli e Maurizio Degli Innocenti, in Brescia 91/97, Documentazione trasmessa dal G.I. di Milano, Fald. G/a-143, Fasc. 3, pp. 27-28.

${ }^{90}$ FIORE 1975, pp. 754-755.

${ }^{91}$ FIORE 1975, pp. 70-71.

${ }_{92}$ Corte di Assise di Appello di Roma, sentenza del 27/11/1984, p. 55.
} 


\section{Processi e rivelazioni}

Nel corso dei primi anni Novanta, alcuni procedimenti importanti, dall'inchiesta "Argo 16", ai nuovi processi su Piazza Fontana e Piazza della Loggia, furono forieri di novità sulla notte di Tora Tora.

Labruna consegnò all'autorità giudiziaria milanese una serie di copie di nastri magnetici, che aveva sino ad allora conservato, contenenti le registrazioni dei suoi colloqui con Orlandini, di cui 8 mai trasmesse alla magistratura, e la registrazione dei due incontri con gli informatori Nicoli e Degli Innocenti. Assieme alle bobine, Labruna aveva esibito al G.I. Guido Salvini una relazione su Avanguardia Nazionale (in realtà già acquisita dalla Commissione P2) e una relazione inedita di Guido Giannettini sul golpe Borghese93. Dalle dichiarazioni di Orlandini «non emersero novità sconvolgenti, ma comunque una serie di elementi che testimoniano la vastità e la profondità dei progetti golpisti e il coinvolgimento e il contatto con un arco di forze assai più ricco di quanto non si sia voluto far credere», come ad esempio l'appoggio di Gelli e della Massoneria italiana ${ }^{94}$. Nei colloqui con Nicoli e Degli Innocenti, emerge in particolare il discorso introduttivo che il colonnello Romagnoli rivolse ai due informatori, nel corso del quale si evidenziava la necessità, da parte del Reparto D, di operare una sorta di "stabilizzazione controllata" dei nuclei eversivi, consegnando alla magistratura le frange più radicali dei vari progetti golpisti, ma nello stesso tempo proteggendo alcuni settori il cui coinvolgimento non doveva assolutamente divenire pubblico. Era necessario evitare, in particolare, che l'autorità giudiziaria di Padova, la quale stava indagando sugli intrecci della destra eversiva veneta, «penetrasse a fondo nelle strutture militari e di sicurezza, toccando santuari - più vicini a un progetto di golpe "bianco" e legale che dovevano assolutamente essere salvaguardati»95. La novità più importante è la presenza del nome di Licio Gelli. Il suo nominativo e il suo ruolo nell'operazione golpista, ovvero la cattura del Presidente della Repubblica Saragat, erano stati infatti omessi nel rapporto consegnato alla magistratura nel 1974. Nell'ottica del colonnello Romagnoli, era certamente doveroso fermare stragi come quella di Brescia che hanno «scosso tutti e non sono sorte spontaneamente per iniziativa di singoli», ma, allo stesso

93 Giornalista legato all'estrema destra e informatore del SID. Indiziato per la strage di Piazza Fontana, venne condannato all'ergastolo nel processo di Catanzaro e in seguito assolto in appello.

94 SALVINI 1995, p. 192; Trascrizione della conversazione del 13 marzo 1973 tra Antonio Labruna e Remo Orlandini, in Brescia 91/97, Documentazione trasmessa dal G.I. di Milano, Fald. G/a-143, Fasc. 2, pp. 104-105.

95 Ibidem, pp. 198-199. 
tempo, si doveva impedire che venissero colpite le «forze sane nell'ambito del Paese, forze che è un peccato che vengano disperse da un'ondata repressiva»96. Allo stesso modo doveva essere evitato di far conoscere la partecipazione di esponenti mafiosi, altro elemento emerso nel corso della stessa conversazione.

Come già accennato, Labruna non aveva solamente consegnato ai magistrati le bobine occultate ma una relazione inedita ad opera di Guido Giannettini. In essa si imputava a Federico Umberto D'Amato di essersi schierato a favore del golpe per poi farlo fallire «e in seguito a ciò condizionare gli ambienti di destra e gli ambienti militari coinvolti nella congiura»97. La nota inoltre elencava coloro i quali avrebbero partecipato attivamente al tentativo golpista, tra i quali l'ammiraglio Giovanni Torrisi, Capo di Stato Maggiore della Marina e in seguito Capo di Stato Maggiore della Difesa. Il suo nome era stato in realtà censurato da Maletti «vista la delicatezza dell'incarico già all'epoca rivestito»98. L'operazione di "sfrondatura" dei nomi più importanti in rapporto al golpe Borghese, non fu soltanto un'iniziativa personale dei vertici dell'Ufficio D, ma venne consigliata dall'allora ministro della Difesa Andreotti. La circostanza venne confermata anche dal colonnello Antonio Viezzer, segretario dell'Ufficio D, oltre che da Labruna99.

Il recente procedimento istruito dalla magistratura di Brescia in seguito alla riapertura delle indagini sulla strage di Piazza della Loggia, ha portato nuove acquisizioni che permettono di ricomporre alcuni tasselli mancanti del golpe Borghese. In particolare vennero acquisite le fotocopie di una lettera dattiloscritta firmata da Borghese, di una lettera datata gennaio 1976 a firma di tale Isla (probabilmente una segretaria del Principe) e di un brogliaccio di appunti dattiloscritti ${ }^{100}$. Sono tre documenti molto interessanti che, se autentici, aiutano a capire meglio il progetto di Borghese, a trovare dei riscontri su persone coinvolte e sui fatti accaduti, ma che pongono ulteriori interrogativi ai quali non è possibile per il momento dare una risposta. Per prima cosa, per quanto riguarda la lettera a firma Borghese, è necessario evidenziare che non è possibile, trattandosi di un testo dattiloscritto, e per giunta fotocopiato, dimostrare inoppugnabilmente la sua autenticità. Tuttavia molti elementi, quali «lo stile letterario compatibile con quello di Borghese, la mancanza di

96 Trascrizione delle conversazioni del 30 maggio 1974 e del 31 maggio 1974 tra Sandro Romagnoli, Maurizio Degli Innocenti, Torquato Nicoli e Antonio Labruna, cit. in ivi, pp. 200202.

${ }^{27}$ Relazione di Guido Giannettini consegnata a Labruna nel 1973; Ibidem, p. 213.

98 Deposizione di Guido Giannettini al G.I. Mastelloni di Venezia, 19 febbraio 1990, in Brescia 91/97, Verbali Milano, Fald. D/b-6, pp. 414-416.

99 Deposizione di Antonio Viezzer al G.I. Mastelloni di Venezia, 18 luglio 1988, in Brescia 91/97 mod. 21, Verbali Brescia, Fald. D/a-21, p. 88.

${ }^{100}$ Per i testi completi vedi All. 1, 2, 3, in GIANNULI n. 50, pp. 7-25. 
anacronismi, errori di nomi, contraddizioni interne al testo e soprattutto la presenza, assieme al testo in questione, della lettera firmata da Isla e del brogliaccio degli appunti, i quali si incrociano perfettamente con la lettera-testamento, plausibilmente portano a far ritenere il documento proveniente almeno da persona vicina se non dallo stesso Borghese»101. Nella lettera il Comandante si rivolgeva ad alcuni ex militari con i quali avrebbe dovuto incontrarsi il 21 e 22 settembre a Nizza per un incontro chiarificatore. Grazie alla lettera di Isla possiamo identificare l'anno con il 1974, pertanto Borghese aveva dettato il testo alla segretaria pochi giorni prima della propria morte $^{102}$. La novità più rilevante consiste nella presenza di Gilberto Bernabei come rappresentante di Giulio Andreotti, indicato dagli americani come "traghettatore" della «drastica svolta della politica nazionale» ${ }^{103}$. Un elemento centrale è la disponibilità, da parte di Borghese, di molti fascicoli «sull'operato dei vari governanti» forniti dall'ex agente dell'OSS James Angleton, Bernabei e Giovanni De Lorenzo, forse documentazione proveniente dal dossieraggio illegale del Sifar ${ }^{104}$. A quanto emerge dalle lettere, gli stessi servizi spagnoli ne avrebbero fatto una copia, prima che gli originali fossero consegnati «al figlio di "B" ", sulla cui identità non è possibile fare ipotesi. Interessanti sono anche i riferimenti dei rapporti intrattenuti con Clavio e con l'ambasciatore statunitense Martin, a conferma dei contatti stretti intrattenuti dai golpisti con emissari degli USA. La parte più ricca di novità è tuttavia il finale della lettera del Principe e dei suoi appunti dove vengono descritte le ultime ore del tentato golpe: il Colonnello Genovesi del SID, venuto a conoscenza della mobilitazione, avrebbe avvertito sia il PCI sia il Presidente della Repubblica Saragat. Miceli, simpatizzando per il golpe, avrebbe tentato di tergiversare ma alla fine si sarebbe arreso a chiamare Bernabei e Clavio. La fuga di notizie, pertanto, avrebbe determinato il ritiro degli americani e il contrordine di "A”, evidentemente Andreotti, per mezzo di Bernabei. La chiusura, inoltre, esplicita la motivazione che ha spinto il Comandante a porre «fine al silenzio». Egli sentiva la necessità di «ristabilire la verità» anche se, avverte, «quanto riportato [...] non potrà certo essere aderente al vero» a causa dei procedimenti

\footnotetext{
${ }^{101}$ GIANNULI, Aldo, op. cit., pp. 129-130.

${ }^{102}$ Vedi GIANNULI n. 50, p. 77.

103 Ibidem, pp. 115-121.

104 Il Servizio Informazione Forze Armate (SIFAR), era il servizio segreto militare precedente al SID. Durante la gestione del Generale De Lorenzo (1955-1962), il Servizio aveva raccolto numerosi dossier su politici, militari, ecclesiastici, giornalisti, sindacalisti, uomini di cultura, andando ben oltre le sue finalità istituzionali. La Camera dei Deputati, in seguito agli esiti una commissione parlamentare di inchiesta, proclamò l'illegalità dei fascicoli e ne stabilì la distruzione. Vedi FRANZINELLI Mimmo, Il piano Solo. I servizi segreti, il centro-sinistra e il "golpe" del 1964, Milano, Mondadori, 2010.
} 
giudiziari in corso, poiché sentiva «che la fine è vicina, naturale o no» ${ }^{105}$. Il vero scopo della lettera era dunque invitare i destinatari a servirsi dell'archivio che avrebbe affidato al figlio di "B" per il suo potenziale intimidatorio. Grazie a questa missiva offriva ai suoi un'arma per difendersi di fronte alle inchieste giudiziarie ma anche nei confronti di coloro i quali, come sosteneva Isla, stavano utilizzando una parte degli stessi fascicoli per ricattare a scopo di lucro. Sicuramente un ulteriore scopo poteva essere individuato nel far sapere ad altri l'esistenza di un suo archivio dal potenziale esplosivo, nel caso la lettera fosse venuta in possesso di qualcun altro al di fuori della cerchia ristretta cui era destinata ${ }^{106}$.

\section{Conclusioni}

Il tentativo di Borghese non fu quel golpe "da operetta" descritto dalla sentenza della Cassazione, né fu velleitario e minoritario. Ad esso presero parte numerosi ufficiali in servizio attivo ed alcuni di grado molto elevato (su tutti Torrisi), esponenti massonici di notevole peso come Licio Gelli, della mafia siciliana e della 'ndrangheta, nonché migliaia di attivisti della destra extraparlamentare in diverse città d'Italia. Si trattava di una situazione intricata nel quale erano in gioco forze contrastanti. Tra queste gli Stati Uniti (ma come abbiamo visto, la loro posizione non era univoca), che da tempo sollecitavano un'azione di forza in modo tale da bloccare l'avanzata comunista, trovando, in tal modo, appoggio in alcune frange dell'esercito italiano. Gli elementi dell'estrema destra che si illudevano veramente di poter ritornare alla ribalta nazionale. I conservatori (destra democristiana, partito liberale, partito socialdemocratico, alcuni ambienti imprenditoriali) i quali pensavano ad un "golpe bianco" di stampo presidenzialista e anticomunista da attuarsi stroncando un tentato colpo di mano fascista, il quale avrebbe giustificato l'utilizzo di un piano di emergenza per mezzo di cui militari e politici centristi sarebbero intervenuti contro le forze sia di destra che di sinistra. Non possono inoltre essere esclusi coloro i quali, come Andreotti e D’Amato, avrebbero collaborato al tentativo per farlo fallire alla prima occasione. Sembra chiaro che Borghese, vista anche la sua reazione alle richieste di spiegazioni in merito all'ordine di fermare l'operazione, fosse consapevole di prestarsi a un golpe "civetta" il cui scopo era unicamente quello di innescare un controgolpe militare. È probabile, visto il contrordine e i suoi sviluppi, che le due fazioni, come le ha definite

105 All. n. 1 in GIANNULI n. 50, p. 8.

${ }^{106}$ Per un'analisi più approfondita dei tre documenti vedi GIANNULI n. 50. 
Aldo Giannuli, dei «doppiogiochisti atlantici» (militari e centristi) e degli «entristi cinici» (Andreotti, D’Amato) avessero trovato un accordo ${ }^{107}$. In questo modo il golpe veniva bloccato, si bruciavano le organizzazioni della destra extraparlamentare ma l'Italia manteneva una linea centrista, conservatrice e filoatlantica. È bene inoltre ricordare il contesto nel quale si erano finalmente avviate le indagini sui golpisti e sulle organizzazioni della destra eversiva. Il 1974 è sì l'anno delle stragi dell'Italicus e di Piazza della Loggia, ma a differenza delle precedenti, significativamente, non si cercò di farne ricadere le responsabilità sui gruppi di sinistra. Esse segnarono il colpo di coda dell'estremismo di destra, braccato dalle indagini della magistratura e falcidiato dagli arresti all'interno di AN e ON. Lo stesso scenario internazionale era cambiato: la presidenza Nixon era investita dallo scandalo Watergate mentre in Europa cadevano le ultime dittature militari di destra, il Portogallo e la Grecia, seguite l'anno successivo dalla Spagna. «Tali cambiamenti», scrive Guido Panvini, «incrinarono ulteriormente la rete di complicità che aveva tenuto assieme settori del mondo militare, del neofascismo e del mondo politico ${ }^{108}$, portando, significativamente, anche all'arresto dell'ex capo del SID Miceli da parte del giudice istruttore di Padova Giovanni Tamburino. Fu sicuramente un cambiamento netto rispetto al passato ma che non durò a lungo. Molte inchieste che avevano coinvolto la destra e gli apparati dello Stato (e come abbiamo visto anche quella sul golpe Borghese) finirono in gran parte con un nulla di fatto. Nello stesso tempo gli equilibri politici tornarono incerti e ripiegarono verso destra, mettendo in definitiva crisi il centro-sinistra.

In conclusione, grazie alle parole di Gaetano Lunetta, uno dei membri di AN che entrarono al Viminale il 7 dicembre, possiamo riassumere al meglio il clima degli anni che seguirono il tentativo di Borghese: «il risultato politico che voleva ottenere chi aveva organizzato l'assalto è stato raggiunto: congelamento della politica di Aldo Moro, allontanamento del PCI dall'area di governo, garanzie di una totale fedeltà filo atlantica e filo americana: la verità è che il golpe c'è stato ed è riuscito» ${ }^{109}$.

107 GIANNULI, Aldo, Il noto servizio, Roma, Castelvecchi, 2013, pp. 129-130.

108 PANVINI, Guido, La destra eversiva, in ORSINA, Giovanni (a cura di), Storia delle destre nell'Italia repubblicana, cit., p. 229.

109 Si veda CUCCHIARELLI, Paolo, GIANNULI, Aldo, Lo Stato parallelo, cit., pp. 257-258. 


\section{* L'autore}

Nicola Tonietto ha conseguito la laurea magistrale in Scienze Storiche presso l'Università di Padova nel 2014. È attualmente dottorando dell'Università di Trieste e Udine con un progetto di ricerca sulle origini del movimento neofascista in Italia.

URL: < http://www.studistorici.com/progett/autori/\#Tonietto >

\section{Per citare questo articolo:}

TONIETTO, Nicola, «Un colpo di stato mancato? II golpe Borghese e l'eversione nera in Italia», Diacronie. Studi di Storia Contemporanea : Stato, costituzione e democrazia, 29/09/2016,

URL:<http://www.studistorici.com/2016/09/29/tonietto_numero_27/ >

\section{Diacronie Studi di Storia Contemporanea $\beta$ www.diacronie.it}

Risorsa digitale indipendente a carattere storiografico. Uscita trimestrale. redazione.diacronie@hotmail.it

Comitato di redazione: Jacopo Bassi - Luca Bufarale - Elisa Grandi - Antonio César Moreno Cantano - Deborah Paci - Fausto Pietrancosta - Alessandro Salvador - Matteo Tomasoni - Luca Zuccolo

Diritti: gli articoli di Diacronie. Studi di Storia Contemporanea sono pubblicati sotto licenza Creative Commons 3.0. Possono essere riprodotti e modificati a patto di indicare eventuali modifiche dei contenuti, di riconoscere la paternità dell'opera e di condividerla allo stesso modo. La citazione di estratti è comunque sempre autorizzata, nei limiti previsti dalla legge. 